\title{
Fatores Sociodemográficos e Ocupacionais Associados à Síndrome de Burnout em Profissionais de Enfermagem
}

\author{
Sociodemographic and Occupational Factors Associated \\ with Burnout Syndrome among Nursing Professionals
}

\author{
Isabella Cristina Moraes Campos ${ }^{*}, a$, Antonio Paulo Angélico ${ }^{b}$, Marcos Santos de Oliveira $^{b}$ \\ \& Daniela Carine Ramires de Oliveira ${ }^{b}$ \\ ${ }^{a}$ Instituto Federal do Sudeste de Minas Gerais, São João del-Rei, MG, Brasil \\ $\&{ }^{b}$ Universidade Federal de São João del-Rei, São João del-Rei, MG, Brasil
}

\begin{abstract}
Resumo
Esta pesquisa de levantamento objetivou investigar a prevalência da síndrome de Burnout nos profissionais de enfermagem de um hospital e de Unidades Básicas de Saúde, comparando-a entre as três categorias profissionais (enfermeiros, técnicos e auxiliares de enfermagem) e os tipos de serviços, e identificou seus fatores sociodemográficos e ocupacionais preditores. A amostra foi composta por 116 profissionais, entre 21 e 60 anos $(M=37,26$ anos; $D P=8,56)$, de ambos os gêneros, de um município de médio porte de Minas Gerais, que responderam ao Inventário de Burnout de Maslach e a um Questionário Sociodemográfico e Ocupacional. Observou-se uma alta prevalência da síndrome (47\%), e um grande número de trabalhadores em risco de adoecimento (entre $41 \%$ a $49 \%$ da amostra, dependendo da dimensão do Burnout). Não houve diferenças significativas em função das categorias profissionais e locais de trabalho. As variáveis ocupacionais "problemas de relacionamento com colegas de trabalho" $(O R=3)$ e "insatisfação com o trabalho" $(O R=11)$ foram as preditoras da síndrome ( $p<$ $0,05)$. As variáveis sociodemográficas não foram significativas. Este estudo contribui para a discussão sobre o processo de desenvolvimento do Burnout em profissionais de enfermagem, ajudando a planejar medidas preventivas de doenças ocupacionais e programas de intervenção nos serviços de saúde.

Palavras-chave: Síndrome de Burnout, Enfermagem, serviços de saúde.
\end{abstract}

\begin{abstract}
This survey research aimed to investigate the prevalence of the Burnout syndrome in nursing professionals of a hospital and Basic Health Units, comparing it among the three professional categories (nurses, nursing technicians and nursing assistants) and types of services, and to identify socio-demographic and occupational factors associated with its occurrence. The sample was composed of 116 professionals, between 21 and 60 years old $(M=37.26$ years; $S D=8.56)$, of both genders, from a medium-sized city of Minas Gerais, who fulfill the Maslach Burnout Inventory and the socio-demographic and occupational questionnaire. There was a high prevalence of the syndrome (47\%) and a large number of workers at risk of illness (between $41 \%$ and $49 \%$ of the sample according to the dimension of Burnout). There were no significant differences between the professional categories and workplaces. The occupational variables "relationship problems with co-workers" $(O R=3)$ and "non-satisfaction with the job" $(O R=11)$ were the syndrome predictors $(p<.05)$. The socio-demographic variables were not significant. This study contributes to the discussion about Burnout developing process among nursing professionals, helping to plan preventive measures of occupational diseases and intervention programs in health services.

Keywords: Burnout syndrome, nursing, health services.
\end{abstract}

Em saúde, o cuidar implica tensão emocional constante, atenção e grandes responsabilidades. A natureza deste trabalho, no qual, às vezes, é necessário lidar com a dor, o sofrimento e a morte de pacientes, pode afetar a saúde dos trabalhadores (França, Ferrari, Ferrari, \& Alves, 2012),

* Endereço para correspondência: Instituto Federal do Sudeste de Minas Gerais, Núcleo de Ambiente, Saúde e Segurança, Rua Patronato, s/n, Vila do Carmo, São João del-Rei, MG, Brasil 36301358.E-mail: isabella.campos@ ifsudestemg.edu.br, angelico@ufsj.edu.br,mso1980@ gmail.com e ramires.daniela@gmail.com causando-lhes prejuízos físicos, sociais e psicológicos (Batista \& Bianchi, 2006).

Dentro deste contexto, a síndrome de Burnout vem sendo considerada um grave problema de saúde pública, acometendo diversos profissionais de saúde. Essa síndrome resulta da vivência profissional em um contexto com muitos estressores emocionais e relações sociais complexas, sendo, portanto, consequente da relação entre o profissional e o seu ambiente de trabalho (Kitze \& Rodrigues, 2008; Lacerda \& Hueb, 2005; Ministério da Saúde, 2001). Desenvolve-se quando as estratégias 
individuais de enfrentamento dos trabalhadores falham ou se tornam insuficientes para lidar com esses agentes estressores (Benevides-Pereira, 2002).

A síndrome foi descrita por Maslach e Jackson (1981) como um conjunto de sintomatologia física e psicológica, sendo constituída por três dimensões relacionadas, mas independentes. A exaustão emocional se relaciona à sensação de esgotamento físico e mental e ao sentimento de falta de energia e entusiasmo. A despersonalização refere-se às alterações na atitude do trabalhador, que começa a ter um contato frio e impessoal com seus clientes ou usuários de seu serviço. A diminuição da realização profissional associa-se ao sentimento de insatisfação quanto às atividades profissionais realizadas, com sentimento de insuficiência, baixa autoestima, fracasso profissional e desmotivação com o trabalho (Benevides-Pereira, 2002; Carlotto \& Câmara, 2008; Codo \& Vasques-Menezes, 1999).

Os enfermeiros, técnicos e auxiliares de enfermagem constituem uma equipe com grande predisposição ao desenvolvimento desta síndrome, por serem os profissionais de saúde que mais tempo passam em contato com o paciente e seus familiares (Carvalho \& Magalhães, 2011). Além disso, no âmbito hospitalar, estes profissionais são, comumente, submetidos a condições insalubres e precárias, que geram uma baixa qualidade de vida no trabalho (Becker \& Oliveira, 2008; Elias \& Navarro, 2006). Somam-se a esses fatores as longas jornadas de trabalho, a rígida estrutura hierárquica, a excessiva carga de trabalho, a execução de ações repetitivas, o número limitado de recursos humanos e materiais, o desgaste psicoemocional nas tarefas realizadas e a pressão de chefias, dos colegas de trabalho e, frequentemente, dos próprios pacientes e familiares (Becker \& Oliveira, 2008; Faria, Barboza, \& Domingos, 2005).

Apesar de os estudos acerca da temática enfatizarem o trabalho em hospitais, os profissionais de enfermagem da atenção primária também enfrentam diversos desafios e estressores laborais, que podem favorecer o desenvolvimento da síndrome de Burnout. Dentre os fatores de risco à saúde desses trabalhadores, estão a exposição à realidade das comunidades onde atuam, nas quais os recursos são escassos, e o contato direto com o cotidiano e o sofrimento do próximo, que, somados às características individuais de cada trabalhador, podem desencadear o processo de estresse e, consequentemente, culminar no desenvolvimento da síndrome. Ademais, os ambientes nos quais esses profissionais atuam são, muitas vezes, perigosos, insalubres e propícios a riscos à saúde (Camelo \& Angerami, 2004; Trindade \& Lautert, 2010).

Em um trabalho de revisão da literatura, foram identificados onze estudos que fizeram o levantamento dos fatores sociodemográficos e ocupacionais relacionados à síndrome de Burnout nos trabalhadores de enfermagem que atuam em hospitais, sendo seis internacionais (Ahmadi, Azizkhani, \& Basravi, 2014; Higashiguchi et al., 1999; Ilhan, Durukan, Taner, Maral, \& Bumin, 2008; Lin, St John, \& McVeigh, 2009; Linde, Martínez, \& Cervantes,
2005; Wang, Liu, \& Wang, 2013) e cinco nacionais (Meneghini, Paz, \& Lautert, 2011; Moreira, Magnago, Sakae, \& Magajewski, 2009; Rissardo \& Gasparino, 2013; Silva \& Carlotto, 2008; Tavares, Souza, Silva, \& Kestenberg, 2014). Apenas um estudo foi realizado com profissionais de Unidades Básicas de Saúde (UBSs) (Holmes, Alves, Holmes, Viana, \& Santos, 2014).

Quanto aos fatores sociodemográficos, três dentre os doze estudos constataram que os profissionais mais jovens tinham maior risco de desenvolver a síndrome (Higashiguchi et al., 1999; Ilhan et al., 2008; Tavares et al., 2014). Por outro lado, três pesquisas apontaram os profissionais mais velhos como os mais suscetíveis ao adoecimento (Holmes et al., 2014; Lin et al., 2009; Linde et al., 2005). Um estudo verificou que as profissionais casadas apresentaram maiores pontuações na subescala de exaustão emocional (Lin et al., 2009). Porém, Tavares et al. (2014) apontaram que ser solteiro(a) era fator determinante para o desenvolvimento da síndrome e outro estudo relatou que pessoas com parceiros fixos tinham menores chances de adoecer (Linde et al., 2005). Quanto à paternidade/maternidade, Linde et al. (2005) verificaram que os profissionais com mais de dois filhos tinham maior realização profissional, enquanto que Higashiguchi et al. (1999) constataram que ter filhos aumentava o risco de exaustão emocional e despersonalização. Por outro lado, Tavares et al. (2014) identificaram a maior susceptibilidade de adoecer nos profissionais sem filhos.

Com relação às variáveis laborais, cinco estudos apontaram que quanto maior era o tempo de serviço em enfermagem, menor era o risco de desenvolver a síndrome (Higashiguchi et al., 1999; Ilhan et al., 2008; Linde et al., 2005; Silva \& Carlotto, 2008; Tavares et al., 2014). Entretanto, dois estudos apresentaram resultados contrários, indicando que quanto maior o tempo de trabalho em enfermagem, maiores foram as pontuações obtidas na subescala de exaustão emocional (Lin et al., 2009; Moreira et al., 2009). Duas pesquisas mostraram que os trabalhadores que atuavam nos setores de internação apresentaram maiores pontuações em despersonalização (Higashiguchi et al., 1999; Linde et al., 2005). Quatro estudos divergiram desse resultado, pois os profissionais com maiores chances de serem acometidos pela síndrome trabalhavam em setores como o Centro de Terapia Intensiva (CTI), Pronto-atendimento e Centro Cirúrgico (Ahmadi et al., 2014; Ilhan et al., 2008; Lin et al., 2009; Tavares et al., 2014). Quanto à carga horária de trabalho, uma pesquisa relatou que quanto maior era o tempo de trabalho, maior era o risco de ocorrência da síndrome (Ilhan et al., 2008). A pesquisa de Silva e Carlotto (2008) contrariou esse resultado, assinalando que a carga horária maior estava associada à menor exaustão emocional e à maior realização profissional. Quanto ao regime de trabalho, dois estudos apontaram que trabalhadores que atuavam em plantões tinham maiores riscos de adoecer (Higashiguchi et al., 1999; Ilhan et al., 2008), enquanto outro estudo indicou que os profissionais do turno da manhã obtiveram maior exaus- 
tão emocional (Linde et al., 2005). Rissardo e Gasparino (2013) verificaram, entretanto, a associação da exaustão emocional com o trabalho noturno.

A análise das pesquisas apontou divergências e contradições quanto às variáveis associadas à síndrome de Burnout e os seus resultados não foram apresentados separadamente por categoria profissional. Verificou-se, também, falta de consenso quanto à interpretação dos dados obtidos com a aplicação do Inventário de Burnout de Maslach (MBI) e variações acerca da realidade social (cultural) e sanitária entre os diferentes países onde os estudos foram realizados.

Com relação à interpretação do MBI, a literatura apresenta dois critérios de rastreamento da síndrome. Ramirez, Graham, Richards, Cull e Gregory (1996) apontaram a sua ocorrência quando houve a combinação simultânea de altas pontuações nas subescalas de exaustão emocional e despersonalização e de baixa pontuação na dimensão de realização profissional. Por outro lado, Grunfeld et al. (2000) empregaram como critério a presença de alta pontuação na subescala de exaustão emocional ou despersonalização ou baixa pontuação na dimensão de realização profissional.

Considerando que a enfermagem constitui uma ocupação com grande predisposição ao desenvolvimento da síndrome de Burnout e a escassez de pesquisas nacionais sobre a temática, este estudo objetivou investigar a prevalência da doença nos profissionais de enfermagem de um hospital e de UBSs, comparar a prevalência entre as três categorias profissionais (enfermeiros, técnicos e auxiliares de enfermagem) e entre os dois tipos de serviços de saúde, e identificar seus fatores sociodemográficos e ocupacionais preditores. Ressalta-se, portanto, como inovação deste estudo, o levantamento da prevalência da síndrome entre os profissionais de UBSs e a comparação desses dados com os de trabalhadores de um hospital.

\section{Método}

\section{Aspectos Éticos}

Foram seguidas as normas da Resolução $\mathrm{n}^{\circ} .466$ de 2012 do Conselho Nacional de Saúde. O desenvolvimento desta pesquisa obteve o consentimento dos responsáveis legais pelas instituições e dos próprios participantes, após aprovação pela Comissão de Ética em Pesquisa Envolvendo Seres Humanos da Universidade Federal de São João del-Rei (Memorando nº. 005/2013/UFSJ/CEPES).

\section{Locais do Estudo}

Este estudo, de abordagem quantitativa, consistiu em uma pesquisa de levantamento, do tipo correlacional e de corte transversal. Foi realizado em um hospital geral sem fins lucrativos, com 123 leitos, onde atuavam 146 profissionais de enfermagem, e em1 8 UBSs, nas quais havia 51 trabalhadores de enfermagem. Essas instituições de saúde situam-se em um município de médio porte do interior de Minas Gerais.

\section{Participantes}

Os critérios de inclusão para a população-alvo foram: idade igual ou superior a 18 anos; pertencer à equipe de enfermagem das instituições; atuar diretamente na assistência aos pacientes há, pelo menos, um ano na mesma instituição; e concordar em participar voluntariamente da pesquisa. Foram excluídos os trabalhadores que estavam de férias ou que estiveram em período de férias nos três meses anteriores à coleta de dados; aqueles afastados das atividades laborais, no momento da coleta, ou que estiveram afastados por tempo igual ou superior a um mês e retornaram ao trabalho em um tempo inferior a três meses; e os trabalhadores que demonstraram dificuldades de compreensão das questões dos instrumentos aplicados.

\section{Instrumentos}

A aplicação dos instrumentos de coleta de dados foi conduzida pela pesquisadora e por três estagiárias em pesquisa, previamente treinadas. Os profissionais que compuseram a amostra responderam, individualmente, aos instrumentos, durante o horário de trabalho. Para avaliar a ocorrência da síndrome de Burnout, foi utilizado o Inventário de Burnout de Maslach - versão Human Services Survey (MBI-HSS). Elaborado por Maslach e Jackson (1981), foi validado para o Brasil por Benevides-Pereira (2001), Lautert (1995) e Tamayo (1997). Possui 22 itens e avalia as três dimensões da síndrome - exaustão emocional, despersonalização e realização profissional -, sendo esta última examinada de forma inversa, ou seja, é considerada a baixa realização profissional (Lautert, 1995; Moreira et al., 2009; Silva \& Carlotto, 2008).

Foi adotada a escala do tipo Likert de 5 pontos, distribuídos em função da frequência, percebida pelo trabalhador, do sentimento ou atitude que estava sendo avaliada, variando de 1 ("nunca") a 5 ("diariamente"); (Carlotto \& Câmara, 2007; Moreira et al., 2009; Silva \& Carlotto, 2008; Tamayo, 1997). Para avaliar a prevalência da síndrome de Burnout, foi empregado o mesmo critério adotado por Grunfeld et al. (2000). A pontuação em cada dimensão da síndrome foi categorizada como: (a) baixa, com percentis $\leq 25$; (b) média, com percentis entre 25 e 75 e (c) alta, com percentis $\geq 75$ (Lautert, 1995).

$\mathrm{O}$ instrumento possui qualidades psicométricas adequadas, tanto de validade quanto de fidedignidade, aferidas por Carlotto e Câmara (2007), em um estudo com uma amostra multifuncional (trabalhadores de instituições de saúde, justiça, segurança e educação), no qual os resultados indicaram uma consistência interna adequada do MBI-HSS, nas suas três dimensões. Os fatores exaustão emocional e realização profissional apresentaram valores de alfa de Cronbach de 0,88 e 0,94 , respectivamente, constituindo-se subescalas com altos índices de consistência interna. $\mathrm{O}$ fator despersonalização obteve um coeficiente alfa inferior $(\alpha=0,65)$, mas próximo do desejado $(\alpha=0,70)$.

No que diz respeito à sua validade, a análise fatorial do instrumento identificou uma estrutura fatorial composta por três fatores, que explicaram $55,68 \%$ da variância dos 
Campos, I. C. M., Angélico, A. P., Oliveira, M. S. \& Oliveira, D. C. R. (2015). Fatores Sociodemográficos e Ocupacionais Associados à Síndrome de Burnout em Profissionais de Enfermagem.

resultados encontrados. Os itens do instrumento apresentaram cargas fatoriais que variaram de 0,43 a 0,89 . Esse inventário foi considerado, portanto, adequado para estudos sobre a síndrome de Burnout (Carlotto \& Câmara, 2007).

O segundo instrumento consistiu em um Questionário Sociodemográfico e Ocupacional elaborado para esta pesquisa e submetido a um estudo piloto. As variáveis que compuseram o instrumento foram selecionadas com base na literatura da área (Benevides-Pereira, 2002; Higashiguchi et al., 1999; Lautert, 1995; Lin et al., 2009; Linde et al., 2005; Meneghini et al., 2011; Moreira et al., 2009; Silva \& Carlotto, 2008). As variáveis sociodemográficas investigadas foram: sexo, idade, ter companheiro(a), escolaridade, renda familiar mensal, renda per capita mensal, ter filho(s), número de filho(s), idade do(s) filho(s) e morar com o(s) filho(s). Foram investigadas as seguintes variáveis ocupacionais: categoria profissional, local e setor de trabalho, tempo de trabalho em enfermagem e na instituição, regime e horário de trabalho, vínculo empregatício, intervalo de tempo desde as últimas férias, frequência com que faz horas-extras, crença de que trabalha em excesso, problemas de relacionamento com colegas, percepção de apoio dos colegas e da chefia imediata, nível de satisfação com o trabalho, afastamento(s) por licença-médica, número de afastamento(s) no último ano e motivo(s), duração do afastamento mais recente, crença de que o trabalho pode causar desgaste físico e mental e que pode trazer riscos, intenção de mudar de profissão, desejo de mudar de emprego se tivesse oportunidade, plano de se aposentar no emprego atual, ter outro emprego ligado à enfermagem, local e carga horária semanal neste trabalho, e exercício de outro emprego ou atividade não ligada à enfermagem, local e carga horária semanal dessa atividade.

\section{Análise dos Dados}

A análise dos dados foi realizada por meio do software Statistical Package for the Social Sciences for Windows (SPSS), versão 13.0. Os testes estatísticos foram conduzidos adotando-se o nível de significância de $p<0,05$. Foi utilizada a estatística descritiva para caracterização da amostra e descrição das pontuações do MBI-HSS. Os dados foram apresentados separadamente em cada subescala, conforme preconizaram Maslach e Jackson (1981).

Para analisar a prevalência da síndrome de Burnout entre os dois locais de trabalho e as três categorias profissionais, foi empregado o Teste Qui-Quadrado. Para a comparação dos grupos caso e não caso da síndrome, em relação aos fatores sociodemográficos e ocupacionais, foi empregado o Teste $t$ de Student para amostras independentes, para variáveis contínuas, e o Teste Qui-Quadrado, para variáveis categóricas.

Foram incluídas, nas análises de regressão logística subequentes, as variáveis que apresentaram diferenças que se mostraram significativas $(p<0,05)$ e as variáveis que apresentaram valores de $p \leq 0,25$, pois poderiam se mostrar significativas nas análises de regressão, segundo recomendações de Hosmer e Lemeshow (2000). Foi empregada a técnica forward stepwise, considerando-se a probabilidade de entrada igual a 0,05 e, de saída, 0,10. A variável dependente foi a ocorrência da síndrome e as variáveis independentes foram as variáveis sociodemográficas e ocupacionais. Para verificação da qualidade do ajuste do modelo de regressão logística, foi calculada a estatística de Hosmer-Lemeshow (Hosmer \& Lemeshow, 2000).

\section{Resultados}

Foi obtida uma amostra de 116 profissionais, do tipo não probabilístico, composta por 37 profissionais das UBSs $(31,9 \%)$ e 79 do hospital $(68,1 \%)$. Esse tamanho amostral permitiu que a amostra tivesse um poder estatístico de, aproximadamente, $88 \%$, com um nível de significância de 5\% (Hines, Montgomery, Goldsman, \& Borror, 2006).

A média de idade dos profissionais foi 37,26 anos (DP $=8,56)$, variando entre 21 e 60 anos, e, dentre os participantes, $87,1 \%$ eram mulheres. Mais da metade dos profissionais tinha companheiro(a) $(58,62 \%)$ e escolaridade inferior à graduação (75\%). A média da renda mensal per capita foi de 1,38 salários mínimos $(D P=1,09)$, sendo que boa parte da amostra (46,03\%) possuía renda per capita mensal média entre um e dois salários mínimos.

A categoria profissional mais numerosa foi a de técnicos de enfermagem $(63,8 \%)$, seguida pela de enfermeiros $(21,5 \%)$ e auxiliares $(14,7 \%)$. Dentre os participantes, $62,9 \%$ consideravam que trabalhavam em excesso e $69,8 \%$ informaram não ter problemas de relacionamento com colegas de trabalho. Além disso, 75\% dos profissionais relataram receber apoio suficiente dos colegas de trabalho e $63,8 \%$ se sentiam apoiados pela chefia imediata, sendo que $65,5 \%$ se consideravam satisfeitos com o trabalho.

Mais da metade dos profissionais $(58,6 \%)$ já se afastou do trabalho por licença-médica e o número médio de afastamentos, no último ano, foi de $1,33(D P=0,71)$. A causa de afastamento mais citada foram as "doenças do sistema osteomuscular e do tecido conjuntivo", que geraram 12 afastamentos, sendo os problemas de coluna os de maior incidência $(n=5)$.

Grande parte dos profissionais considerou que o trabalho poderia gerar desgaste físico e mental $(87,9 \%)$. Um terço dos trabalhadores $(33,6 \%)$ pensava em mudar de profissão e a maioria apontou que, se tivesse oportunidade, mudaria de emprego $(68,1 \%)$. Apenas $18,11 \%$ dos profissionais tinham mais de um emprego ligado à enfermagem, com carga horária semanal média de 28,24 horas $(D P=15,79)$ nesse emprego. Por outro lado, 13,8\% dos participantes tinham outro emprego ou atividade não ligada à enfermagem, com carga horária semanal média de 16,21 horas $(D P=14,79)$ nessa atividade.

Segundo o critério adotado por Grunfeld et al. (2000), $46,6 \%(n=54)$ dos profissionais satisfizeram os critérios de rastreamento diagnóstico para a síndrome de Burnout. Empregando-se o Teste Qui-Quadrado, não houve diferença significativa entre o número de participantes da amostra total classificados como casos e não casos da síndrome, $\chi^{2}$ $=0,55, p=0,458$. As médias das pontuações por subescala e a distribuição dos profissionais, segundo a classificação das pontuações obtidas, estão apresentadas na Tabela 1. 
Psychology/Psicologia: Reflexão e Crítica, 28(4), 764-771.

Tabela 1

Distribuição das Médias, Desvios-padrão e Nivel de Classificação das Pontuações do MBI-HSS por Subescala (N=116)

\begin{tabular}{lccc}
\hline \multicolumn{1}{c}{ Subescalas } & Médias $(D P)$ & Níveis de classificação & $n / \%$ \\
\hline \multirow{2}{*}{ Exaustão Emocional } & & Alta & $30(25,90 \%)$ \\
& $23,67(7,30)$ & Média & $57(49,10 \%)$ \\
& & Baixa & $29(25,00 \%)$ \\
Despersonalização & & Alta & $29(25,00 \%)$ \\
& & Média & $48(41,40 \%)$ \\
Realização Profissional & Baixa & $39(33,60 \%)$ \\
& & Alta & $31(26,70 \%)$ \\
& $33,96(5,03)$ & Média & $55(47,40 \%)$ \\
& & Baixa & $30(25,90 \%)$ \\
\hline
\end{tabular}

Nota. $D P=$ desvio-padrão; $n=$ número de participantes; $\%=$ percentagem.

A categoria profissional que obteve a maior percentagem de acometidos pela síndrome de Burnout foi a de auxiliares de enfermagem $(52,94 \%)$, quando comparada à de técnicos $(50 \%)$ e enfermeiros ( $32 \%$ ), embora não fosse constatada diferença significativa da prevalência entre as três categorias $\left(\chi_{(1)}^{2}=2,76, p=0,252\right)$.

Com relação ao local de trabalho, um percentual maior de profissionais do hospital apresentou indicativos de exaustão emocional (26,58\%), despersonalização $(25,32 \%)$ e baixa realização profissional (30,38\%), comparativamente aos profissionais das UBSs $(24,32 \% ; 24,32 \%$ e $16,21 \%$, respectivamente). $\mathrm{O}$ maior percentual de trabalhadores rastreados com a síndrome $(48,1 \%)$ foi observado no hospital, em comparação às UBSs $(43,24 \%)$. Todavia, também não foi verificada diferença significativa entre os dois locais do estudo $\left(\chi_{(1)}^{2}=0,24 ; p=0,625\right)$.

Na comparação dos grupos caso e não caso de Burnout, em relação aos fatores ocupacionais e sociodemográficos, as variáveis ocupacionais que apresentaram valores de $p \leq 0,25$ foram: número de afastamentos no último ano $\left(t_{(34)}=-1,84\right)$, carga horária em outro emprego ligado à enfermagem $\left(t_{(19)}=-2,08\right)$, carga horária em outro emprego ou atividade não ligada à enfermagem $\left(t_{(12)}=-2,00\right)$, crença que trabalha em excesso $\left(\chi_{(1)}^{2}=5,38\right)$, problemas de relacionamento com colegas de trabalho $\left(\chi_{(1)}^{2}=9,77\right)$, apoio de colegas de trabalho $\left(\chi_{(1)}^{2}=5,59\right)$, apoio da chefia imediata $\left(\chi_{(1)}^{2}=16,38\right)$, satisfação com o trabalho $\left(\chi_{(1)}^{2}\right.$ $=31,71)$, crença de que o trabalho pode causar desgaste físico e mental $\left(\chi_{(1)}^{2}=4,04\right)$, planos de mudar de profissão $\left(\chi_{(1)}^{2}=9,55\right)$, desejo de mudar de emprego se tivesse oportunidade $\left(\chi_{(1)}^{2}=2,85\right)$, e exercício de alguma atividade ou emprego não ligado à enfermagem $\left(\chi_{(1)}^{2}=1,75\right)$. Por sua vez, as variáveis sociodemográficas com valores de $p \leq$ 0,25 foram: escolaridade $\left(\chi_{(1)}^{2}=3,74\right)$, e renda per capita mensal $\left(t_{(111)}=1,32\right)$.

A princípio, foram feitas análises de regressão logística apenas com as variáveis ocupacionais com valores de $p$ $\leq 0,25$, visto que a literatura destaca a importância do contato do indivíduo com o mundo profissional no desenvolvimento da síndrome de Burnout (Benevides-Pereira, 2002; Kitze \& Rodrigues, 2008; Lacerda \& Hueb, 2005; Ministério da Saúde, 2001). Os resultados referentes ao modelo final, no qual permaneceram as variáveis "problemas de relacionamento com colegas de trabalho" $(0=$ não e $1=\operatorname{sim})$ e "satisfação com o trabalho" $(0=$ satisfeito e 1 $=$ insatisfeito) estão apresentados na Tabela 2.

Tabela 2

Coeficientes Estimados para o Modelo de Regressão Logística, Obtidos a Partir das Variáveis Ocupacionais Significativas

\begin{tabular}{lcccccc}
\hline Variáveis Preditoras & Beta & EP & Wald & $p$ & OR & IC \\
\hline Constante & $-1,24$ & 0,30 & 17,16 & 0,001 & 0,29 & - \\
$\begin{array}{l}\text { Problemas de relacionamento com } \\
\text { colegas de trabalho }\end{array}$ & 1,06 & 0,49 & 4,67 & 0,031 & 2,90 & {$[1,10 ; 7,61]$} \\
\begin{tabular}{l} 
Satisfação com o trabalho \\
\hline
\end{tabular} & 2,40 & 0,50 & 23,22 & 0,001 & 11,04 & {$[4,16 ; 29,30]$} \\
\hline
\end{tabular}

Nota . EP = erro padrão; $p=$ nível de significância; $O R=$ Odds Ratio $; \mathrm{IC}=$ intervalo de confiança. 
Campos, I. C. M., Angélico, A. P., Oliveira, M. S. \& Oliveira, D. C. R. (2015). Fatores Sociodemográficos e Ocupacionais Associados à Síndrome de Burnout em Profissionais de Enfermagem.

O valor da estatística de Hosmer-Lemeshow foi 0,07 , e o correspondente nível descritivo ( $p$-value), calculado a partir da distribuição Qui-Quadrado com 2 graus de liberdade, foi 0,964 , indicando que o referido modelo apresentou um bom ajuste. $\mathrm{O}$ valor de $R^{2}$ de Cox e Snell foi igual a 0,28 , apontando que $28 \%$ das variações ocorridas na razão de chances foram explicadas pelo conjunto das variáveis que se mostraram significativas para a previsão da síndrome de Burnout. É possível afirmar que os profissionais que relataram problemas de relacionamento com colegas de trabalho apresentaram uma chance 2,90 maior de adoecer em relação àqueles que não referiram os mesmos problemas. Os trabalhadores que relataram insatisfação com o trabalho obtiveram uma chance 11,04 maior de adoecer comparativamente aos satisfeitos.

No segundo modelo de regressão logística, foram mantidas as variáveis ocupacionais identificadas como importantes para predizer a síndrome e as variáveis sociodemográficas "escolaridade" e "renda per capita mensal" para verificar o quanto essas variáveis influenciariam na ocorrência da síndrome. Ambas variáveis não se mostraram significativas, $\operatorname{com} p=0,914$ e 0,216 , respectivamente.

\section{Discussão}

Neste estudo, a prevalência da síndrome de Burnout mostrou-se elevada, acometendo quase metade da amostra. Considerando-se o grande número de profissionais de enfermagem que estão no mercado de trabalho, supõe-se que muitas pessoas estejam vulneráveis à síndrome. Para Carvalho e Magalhães (2011), os profissionais de enfermagem têm grande predisposição ao desenvolvimento da síndrome por, dentre outros fatores, serem os profissionais de saúde que mais tempo passam em contato com o paciente e seus familiares e, consequentemente, com maior risco de adoecimento.

Essa constatação é relevante, principalmente, quando a esses dados são somados o grande número de trabalhadores que se mostrou vulnerável ao desenvolvimento da síndrome, ou seja, aqueles que obtiveram pontuações médias nas subescalas do MBI-HSS. Neste caso, os estressores ocupacionais, se persistentes, podem acarretar a síndrome de Burnout (Silva \& Carlotto, 2008), pois a doença se desenvolve quando as estratégias individuais de enfrentamento falham ou se tornam insuficientes (Benevides-Pereira, 2002).

Na literatura analisada, os dados de prevalência da síndrome de Burnout não foram divididos entre as categorias profissionais. Nesta pesquisa, como não houve diferença significativa entre a prevalência nas três categorias, pode-se afirmar que, apesar de desenvolverem funções distintas, os trabalhadores tinham o mesmo risco de desenvolver a doença, independentemente da categoria profissional a qual pertenciam.

Com relação ao local de trabalho, um percentual maior de profissionais do hospital apresentou indicativos de exaustão emocional, despersonalização e baixa realização profissional, comparativamente àquele obtido pelos profissionais das UBSs. Contudo, não houve diferença significativa da prevalência entre os dois locais do estudo e, por conseguinte, pode-se afirmar que os profissionais que atuavam em ambos os tipos de serviços de saúde estariam sujeitos ao adoecimento, embora houvesse diferenças entre as atividades desenvolvidas, a organização e as condições de trabalho, dentre outras características que diferenciam o trabalho nas duas instituições de saúde.

A variável "problemas de relacionamento com colegas de trabalho" foi apontada como uma das preditoras da síndrome dentre os profissionais que compuseram a amostra, comprovando que boas relações no trabalho são importantes para a saúde dos profissionais. Silva e Carlotto (2008) verificaram que a insatisfação com as relações hierárquicas esteve significativamente relacionada às três dimensões da síndrome $(p<0,001)$. Em seu estudo, Ilhan et al. (2008) afirmaram que a insatisfação nas relações sociais no trabalho influenciou no desenvolvimento da síndrome, pois os trabalhadores poderiam se sentir excluídos e solitários, não tendo com quem compartilhar frustrações e não recebendo feedbacks positivos de seus superiores. Benevides-Pereira (2002) também considerou o relacionamento entre colegas de trabalho como fator que poderia influenciar no surgimento da síndrome. Além disso, Ilhan et al. (2008) apontaram o apoio social com um fator protetor da síndrome de Burnout.

Esta pesquisa também comprovou que a insatisfação com o trabalho foi uma das variáveis preditoras da síndrome. No estudo de Silva e Carlotto (2008), a satisfação intrínseca com o trabalho e oportunidade de crescimento estiveram significativamente associadas à exaustão emocional e à realização profissional $(p<0,001)$. Em sua pesquisa, Ruviaro e Bardagi (2010) também apontaram a existência de relações entre as dimensões da síndrome e a satisfação com o trabalho $(p<0,05)$.

Os resultados deste estudo confirmaram que as variáveis ocupacionais são fatores desencadeadores da síndrome. Esses fatores estressores envolvem aspectos da organização, da administração, do sistema do trabalho e das relações humanas (Costa, Lima, \& Almeida, 2003).

Esses resultados contribuem para ampliar o conhecimento existente na área e servem como fonte de consulta, com vistas à melhoria da qualidade de vida no trabalho de profissionais da equipe de enfermagem. Neste sentido, poderão subsidiar esforços para que se alcance uma forma de organização do trabalho que minimize seus possíveis efeitos nocivos à saúde desses trabalhadores. Ademais, torna-se necessário um melhor preparo das instituições de saúde para a identificação precoce da síndrome, visando ao planejamento de estratégias preventivas e de seu tratamento, tendo em vista que pode acometer um número considerável de profissionais.

Entretanto, uma limitação do presente estudo consiste na impossibilidade de se generalizar os seus resultados 
para outras populações, suscitando a necessidade de realização de novas pesquisas com amostras mais amplas e em diferentes contextos e regiões brasileiras. Outro fator que impede a generalização dos resultados foi o emprego de percentis para avaliar os resultados do MBI-HSS, pois os valores de percentis de cada subescala foram calculados especificamente para a amostra desse estudo. Essa técnica teve que ser adotada devido à falta de uma amostra normativa nacional e de consenso na literatura da forma de interpretação dos resultados do MBI-HSS, o que dificulta muito a sua utilização.

A grande dificuldade da escala percentílica reside no fato de ser uma escala ordinal, ou seja, não possui valor constante, representando apenas a posição relativa ou ordinal de cada indivíduo dentro do seu próprio grupo. Por esse motivo, não permite o cálculo da média, desvio-padrão e outras medidas estatísticas deles decorrentes (Erthal, 2003). Desse modo, as distâncias entre escores sucessivos não são constantes, mas variam de acordo com a posição do escore, que pode estar no início, meio ou fim da escala, implicando que diferenças iguais entre percentis não significam diferenças iguais em termos dos escores brutos (Pasquali, 2003). No entanto, o fato da escala percentílica representar a posição relativa de cada sujeito dentro do seu próprio grupo confere uma maior validade interna aos dados de estudos que a empregam.

Conforme Contandriopoulos, Champagne, Potvin, Denis e Boyle (1994) e Selltiz, Wrightsman e Cook (1987) afirmaram, por esta pesquisa ser do tipo correlacional, não se pode garantir uma relação de causalidade entre os fatores associados, como as variáveis ocupacionais, e a ocorrência da síndrome de Burnout nos trabalhadores. Desse modo, foram investigadas apenas as possíveis relações existentes entre essas variáveis e a síndrome.

\section{Conclusão}

Os dados verificados são preocupantes, tendo em vista a alta prevalência da síndrome entre os profissionais que compuseram a amostra deste estudo. Os resultados obtidos com a realização dessa pesquisa possibilitaram identificar quais variáveis estiveram relacionadas à ocorrência da síndrome de Burnout nos profissionais de enfermagem, possibilitando, assim, uma reflexão sobre o processo de adoecimento dos profissionais de enfermagem que atuam nos hospitais e nas UBSs.

As pesquisas sobre a síndrome de Burnout, tanto as internacionais quanto as nacionais, sempre focaram os trabalhadores que atuavam nos hospitais e, no entanto, os resultados desse estudo demonstraram não haver diferença significativa na prevalência da síndrome entre o hospital e as UBSs. Tendo em vista a complexidade do trabalho em enfermagem e as diferentes situações em que os profissionais atuam, seria pertinente caracterizar melhor os fatores associados à ocorrência da síndrome em distintas instituições a partir da realização de mais pesquisas na área.

\section{Referências}

Ahmadi, O., Azizkhani, R., \& Basravi, M. (2014). Correlation between workplace and occupational burnout syndrome in nurses. Advanced Biomedical Research, 3(1), 44. doi:10.4103/2277-9175.125751

Batista, K. M., \& Bianchi, E. R. F. (2006). Estresse do enfermeiro em unidade de emergência. Revista Latino-americana de Enfermagem, 14(4), 534-539. doi:10.1590/ S0104-11692006000400010

Becker, S. G., \& Oliveira, M. L. C. (2008). Estudo do absenteísmo dos profissionais de enfermagem de um centro psiquiátrico em Manaus, Brasil. Revista Latino-Americana de Enfermagem 16(1), 109-114. doi:10.1590/S0104-11692008000100017

Benevides-Pereira, A. M. T. (2001). MBI - Maslach Burnout Inventory e suas adaptações para o Brasil. In Anais da XXXII Reunião Anual de Psicologia (pp. 84-85). Rio de Janeiro, RJ: Sociedade Brasileira de Psicologia.

Benevides-Pereira, A. M. T. (2002). Burnout: O processo de adoecer pelo trabalho. In A. M. T. Benevides-Pereira (Ed.), Burnout: Quando o trabalho ameaça o bem-estar do trabalhador (pp. 21-91). São Paulo, SP: Casa do Psicólogo.

Camelo, S. H. H., \& Angerami, E. L. S. (2004). Sintomas de estresse nos trabalhadores atuantes em cinco núcleos de saúde da família. Revista Latino-Americana de Enfermagem, 12(1), 14-21. doi:10.1590/S0104-11692004000100003

Carlotto, M. S., \& Câmara, S. G. (2007). Propriedades psicométricas do Maslach Burnout Inventory em uma amostra multifuncional. Estudos de Psicologia (Campinas), 24(3), 325-332. doi:10.1590/S0103-166X2007000300004

Carlotto, M. S., \& Câmara, S. G. (2008). Análise da produção científica sobre a síndrome de burnout no Brasil. Psico (Porto Alegre), 39(2), 152-158. Recuperado em http://revistaseletronicas.pucrs.br/ojs/index.php/revistapsico/article/view/1461

Carvalho, C. G., \& Magalhães, S. R. (2011). Síndrome de burnout e suas consequências nos profissionais de enfermagem. Revista da Universidade Vale do Rio Verde, 9(1), 200-210. doi:10.5892/ruvrd.v9i1.86

Codo, W., \& Vasques-Menezes, I. (1999). O que é burnout? In W. Codo (Ed.), Educação: Carinho e trabalho (pp. 237-254). Petrópolis, RJ: Vozes.

Contandriopoulos, A. P., Champagne, F., Potvin, L., Denis, J. L., \& Boyle, P. (1994). Saber preparar uma pesquisa. São Paulo, SP: Hucitec Abrasco.

Costa, J. R. A., Lima, J. V., \& Almeida, P. C. (2003). Stress no trabalho do enfermeiro. Revista da Escola de Enfermagem da USP, 37(3), 63-71. doi:10.1590/S0080-62342003000300008

Elias, M. A., \& Navarro, V. L. (2006). A relação entre o trabalho, a saúde e as condições de vida: Negatividade e positividade no trabalho das profissionais de enfermagem de um hospital escola. Revista Latino-Americana de Enfermagem, 14(4), 517-525. doi:10.1590/S0104-11692006000400008

Erthal, T. C. (2003). Manual de Psicometria (7. ed.). Rio de Janeiro, RJ: Jorge Zahar.

Faria, A. C., Barboza, D. B., \& Domingos, N. A. M. (2005). Absenteísmo por transtornos mentais na enfermagem no período de 1995 a 2004. Arquivos de Ciências da Saúde, 12(1), 14-20. doi:10.1590/S1983-14472010000100022

França, F. M., Ferrari, R., Ferrari, D. C., \& Alves, E. D. (2012). Burnout e os aspectos laborais na equipe de enfermagem de dois hospitais de médio porte. Revista Latino-Americana de Enfermagem, 20(5), 961-970. doi:10.1590/S010411692012000500019 
Campos, I. C. M., Angélico, A. P., Oliveira, M. S. \& Oliveira, D. C. R. (2015). Fatores Sociodemográficos e Ocupacionais Associados à Síndrome de Burnout em Profissionais de Enfermagem.

Grunfeld, E., Whelan, T. J., Zitzelsberger, L., Willan, A. R., Montesanto, B., \& Evans, W. K. (2000). Cancer care workers in Ontario: Prevalence of burnout, job stress and job satisfaction. Canadian Medical Association Journal, 163(2), 166-169.

Higashiguchi, K., Morikawa, Y., Miura, K., Nishijo, M., Tabata, M., Ishizaki, M., \& Nakagawa, H. (1999). Burnout and related factors among hospital nurses. Journal of Occupational Health, 41(4), 215-224.

Hines, W. W., Montgomery, D. C., Goldsman, D. M., \& Borror, C. M. (2006). Probabilidade e estatística na Engenharia (4. ed). Rio de Janeiro, RJ: LTC.

Holmes, E., Alves, J., Holmes, D., Viana, Y., \& Santos, S. (2014). Síndrome de burnout em enfermeiros da Estratégia Saúde da Família. Revista de Enfermagem UFPE, 8(7), 1841-1847. doi:10.5205/reuol.5963-51246-1-RV.0807201402

Hosmer, D. W., \& Lemeshow, S. (2000). Applied logistic regression $\left(2^{\text {nd }}\right.$ ed). New York: John Wiley and Sons.

Ilhan, M. N., Durukan, E., Taner, E., Maral, I., \& Bumin, M. A. (2008). Burnout and its correlates among nursing staff: Questionnaire survey. Journal of Advanced Nursing, 61(1), 100-106.

Kitze, S., \& Rodrigues, A. B. (2008). Burnout em oncologia: Um estudo com profissionais de enfermagem. Einstein, 6(2), 128-133.

Lacerda, P. N., \& Hueb, F. D. (2005). A avaliação da síndrome de burnout com enfermeiros de um hospital geral. Revista da Sociedade de Psicologia do Triângulo Mineiro, 9(1), 102-109.

Lautert, L. (1995). O desgaste profissional do enfermeiro (Tese de doutorado, Universidade Pontífica de Salamanca, Espanha).

Lin, F., St John, W., \& McVeigh, C. (2009). Burnout among hospital nurses in China. Journal of Nursing Management, 17(3), 294-301. doi:10.1111/j.1365-2834.2008.00914.x

Linde, J. M. M., Martínez, F. A., \& Cervantes, I. G. (2005). Burnout en enfermería de atención hospitalaria. Enfermería Clínica, 15(5), 275-282.

Maslach, C., \& Jackson, S. E. (1981). The measurement of experienced burnout. Journal of Organizational Behavior, 2(2), 99-113.

Meneghini, F., Paz, A. A., \& Lautert, L. (2011). Fatores ocupacionais associados aos componentes da síndrome de burnout em trabalhadores de enfermagem. Texto e Contexto em Enfermagem, 20(2), 225-233. doi:10.1590/S010407072011000200002

Ministério da Saúde. (2001). Doenças relacionadas ao trabalho: Manual de procedimentos para os serviços de saúde. Brasília, DF: Autor.

Moreira, D. S., Magnago, R. F., Sakae, T. M., \& Magajewski, F. R. L. (2009). Prevalência da síndrome de burnout em trabalhadores de enfermagem de um hospital de grande porte da região sul do Brasil. Cadernos de Saúde Pública, 25(7), 1559-1568. doi:10.1590/S0102-311X2009000700014

Pasquali, L. (2003). Psicometria: Teoria dos testes na psicologia e na educação. Petrópolis, RJ: Vozes.

Ramirez, A. J., Graham, J., Richards, M. A., Cull, A., \& Gregory, W. M. (1996). Mental health of hospital consultants: The effects of stress and satisfaction at work. Lancet, 347(9003), 724-728.

Rissardo, M., \& Gasparino, R. (2013). Exaustão emocional em enfermeiros de um hospital público. Escola Anna Nery, 17(1), 128-132. doi:10.1590/S1414-81452013000100018

Ruviaro, M. F. S., \& Bardagi, M. P. (2010). Síndrome de burnout e satisfação no trabalho em profissionais da área de enfermagem do interior do RS. Barbarói, 33, 194-216.
Selltiz, C., Wrightsman, L. S., \& Cook, S. W. (1987). Métodos de pesquisa nas relações sociais: Delineamentos de pesquisa (2. ed., Vol. 2). São Paulo, SP: Editora Pedagógica e Universitária.

Silva, T. D., \& Carlotto, M. S. (2008). Síndrome de burnout em trabalhadores da enfermagem de um hospital geral. Revista da Sociedade Brasileira de Psicologia Hospitalar, 11(1), 113-130.

Tamayo, M. R. (1997). Relação entre a síndrome de burnout e os valores organizacionais no pessoal de enfermagem de dois serviços públicos (Dissertação de mestrado, Universidade Federal de Brasília, DF, Brasil).

Tavares, K., Sousa, N., Silva, L., \& Kestenberg, C. (2014). Ocorrência da síndrome de Burnout em enfermeiros residentes. Acta Paulista de Enfermagem, 27(3), 260-265. doi:10.1590/1982-0194201400044

Trindade, L. L., \& Lautert, L. (2010). Síndrome de burnout entre os trabalhadores da Estratégia de Saúde da Família. Revista da Escola de Enfermagem da USP, 44(2), 274-279. doi:10.1590/ S0080-62342010000200005

Wang, S. S., Liu, Y. H., \& Wang, L. L. (2013). Nurse burnout: Personal and environmental factors as predictors. International Journal of Nursing Practice. Advance online publication. doi:10.1111/ijn.12216 